\title{
MacLaboratory Controller: A switch and A/D interface between Apple's Macintosh and peripheral apparatus
}

\author{
DOUGLAS L. CHUTE, DARRYL GAEMAN, and TERRY ZIEGLER \\ Drexel University, Philadelphia, Pennsylvania
}

\begin{abstract}
Apple's Macintosh microcomputer has advantages over other systems used for laboratory control and data acquisition, especially because of its graphic and user-friendly features. However, a major perceived limitation has been its closed architecture. We describe an interface using the RS 422 modem port that supports up to 64 input/output $(\mathrm{I} / \mathrm{O})$ functions including analog-to-digital 4-12 channel input. The Controller software supplies an easily edited authoring tool in which functional relationships, logical operators, timing (millisecond accuracy), and counting functions are established through simple mouse and menu commands. Data from I/O functions may be displayed or saved as tab-delimited files that can be opened by various Macintosh statistical and spreadsheet packages and that are transportable to some mainframe applications.
\end{abstract}

One of the perceived limitations of the Macintosh as a controlling device for the laboratory has been its closed architecture. Not wishing to wait for the open architecture version with the hope that some manufacturer of circuit boards would address the need, we have designed and constructed an external controller to support both switched inputs and outputs and analog-to-digital (A/D) conversion. The primary design concern was that the system should provide the user with maximum flexibility by providing an authoring tool that is totally independent of any knowledge of or skill in computer programming, per se. For teaching purposes, our overall approach has been to use the microcomputer as a tool and not as a tutor or tutee (see Hewett, 1986).

In our experience, systems for laboratory control using the IBM PC or Apple II environments required so much programming support and experience that colleagues and students, from the postdoctoral level down to the undergraduate level, avoided using them. If a routine was developed, the difficulties associated with modification functionally tended to lock the microcomputer into a dedicated system. Data that are collected often are not readily available for transportation to data analysis software. In addition, fairly simple relay-only systems that may be purchased cost at least $\$ 2,000$ for the basics, excluding the computer (e.g., Digitry Company, Inc.'s Cognitive Testing Station, 1985). In our experience, the worst situation that emerged was that faculty with sufficiently sophisticated programming skills to operate these systems at the research level were often unwillingly coerced into being technicians in the service of their colleagues and students. Teaching also can suffer because of the high "cost" of

Software development was supported in part by grants to Douglas $\mathrm{L}$. Chute from Apple Computers, Inc., and from the Pew Memorial Trust. Our special thanks to J. Timoteo of the Drexel University Software Development Group. Send reprint requests to Douglas L. Chute, Director of Neuropsychology, Drexel University, Philadelphia, PA 19104. generating software and pedagogical materials, such as laboratory manuals and documentation, for such systems. In general, individuals who are interested in computer applications often find that they can be scientists or programmers, but seldom both. Academic environments are reluctant to recognize or reward the programmers. In effect, these traditional problems created the set of design goals that we set out to satisfy.

\section{GENERAL DESCRIPTION}

MacLaboratory Controller is a software application that establishes user-edited wiring, timing, counting, and logical relationships between inputs and outputs. An Apple Macintosh 512e or Macintosh Plus (required for A/D option), with System v3.2 and Finder v5.3 or later, is necessary. An $800 \mathrm{~K}$ external drive is recommended. The hardware interface using the RS-422 modem port consists of commercially available components and boards that theoretically can provide for up to 255 inputs and outputs (I/O), which can include up to 12 channels of $A / D$ input. We recommend no more than $64 \mathrm{I} / \mathrm{O}$ functions. Various configurations of relay switches (in blocks of eight) and A/D channels can be specified. Although the Controller was designed to support the undergraduate teaching program in psychology (Chute, 1986a, 1986b; Chute \& Daniel, 1986a, 1986b), it can accept any input or drive any output that the user has configured to be compatible with the hardware interface. The cost depends upon the user-specified options.

\section{Software}

The software component employs the general features of the Macintosh standard user interface. To create an experiment file, the user wires-up the various functions and relationships desired. By using the mouse to click and drag, wires, timers, counters, and clocks are connected 


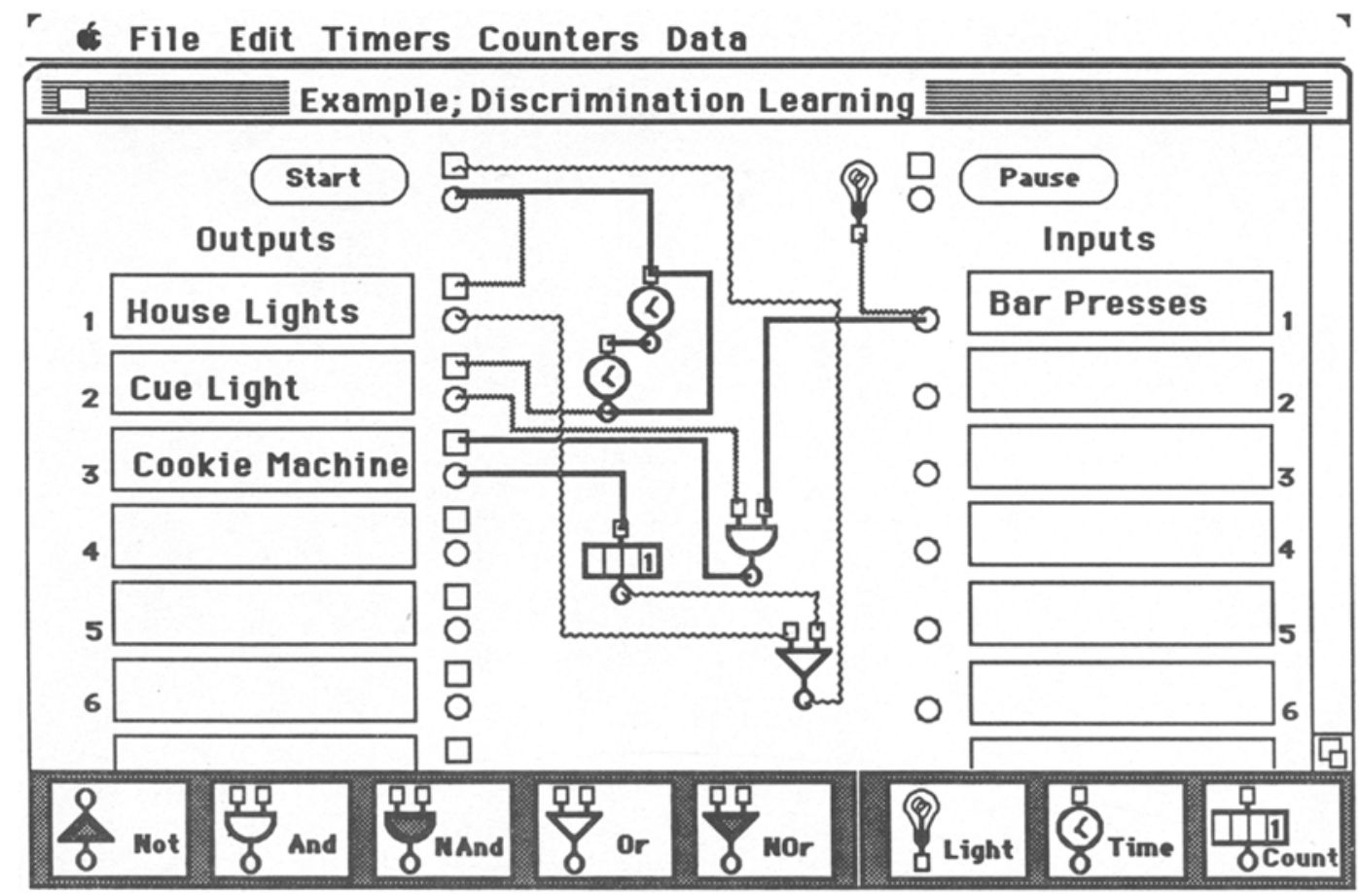

Figure 1. A screen dump of a simple experiment file. By clicking and dragging with the mouse, the experimenter selects and "wires-up" various inputs and outputs to the interface with internal operations selected from the palette. In this example, clicking on the start button brings on the house lights, and the timer pair alternates periods when the discrimination cue light is present. The And gate permits only those barpresses that occur during the cue to be reinforced. The experiment ends either if the timer set in the house lights times out or if the count-down counter reaches a preset number of reinforcements.

on the screen in a manner analogous to setting up relay or solid-state circuitry devices. Within the limits of the Macintosh environment, any number of experiment files can be created and saved with their various wiring and logical operator arrangements. Figure 1 shows a screen dump of a simple experiment file, in this case, one that would control a Skinner box for a discrimination learning task.

In setting up an experiment file, the user specifies which data or events are to be stored in data files. In the case of switch inputs, counting and/or clock/timing data are stored as tab-delimited files that can be opened by most Macintosh statistical, graphic, and spreadsheet programs, or transferred fairly readily as ASCII code to mainframes (see Lehman, 1986). As an example, Figure 2 illustrates the data display window from the short discrimination learning experiment.

When $A / D$ input data is received, it is stored for the time and duration selected, as a tab-delimited file, within the constraints of available memory. This file may be displayed and scrolled in oscilloscope fashion from within the application where time scale and amplitude can be adjusted (Figure 3). The standard A/D option provides for 4 channels, each with a sampling rate of 1024 bytes per second. Practical limitations require disk storage of $A / D$ data after $60 \mathrm{sec}$ of sampling duration. The handling of real-time analog data display is not currently supported by the MacLaboratory Controller. A Macintosh-based system developed at Reed College (Schlickheiser, 1986) does handle real-time displays, but it requires considerable programming support.

Timing routines are established to millisecond accuracy by software clocks or timers (Westall, Perkey, \& Chute, 1986). However, the devices attached by the user to the interface may have their own response latencies, so if timing is crucial, those devices are the limiting factor. Since timing can be affected by writing to disk, data register storage to disk is under program or user control so that timing accuracy is maintained. Timers or clocks can be set by selecting either an input or an output box, or by moving a timer from the palette and placing it in an internal logic circuit. Timers can either record duration or time down from a preset value. They pass a signal to succeeding logic circuits either when they start, during timing (time in), or after time has expired (time out). Figure 4 displays a timing window. Counters can both count events and record the time at which a count occurs. Presetting a value allows a counter to count down before passing a signal on to succeeding logic circuits.

\section{Hardware}

The core component is the Micromint BASIC-52 Computer/Controller from Micromint, Inc. (Vernon, CT). This single-board computer contains an Intel 8052AH- 


\section{File Edit Timers Counters Data}

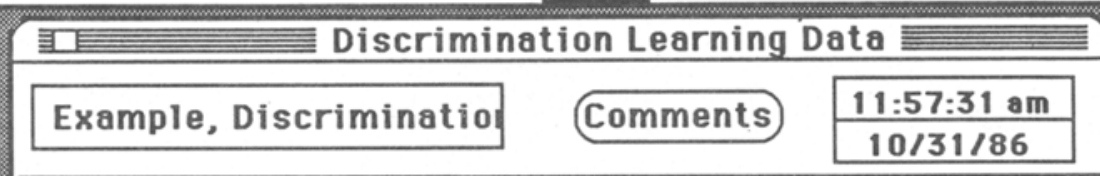

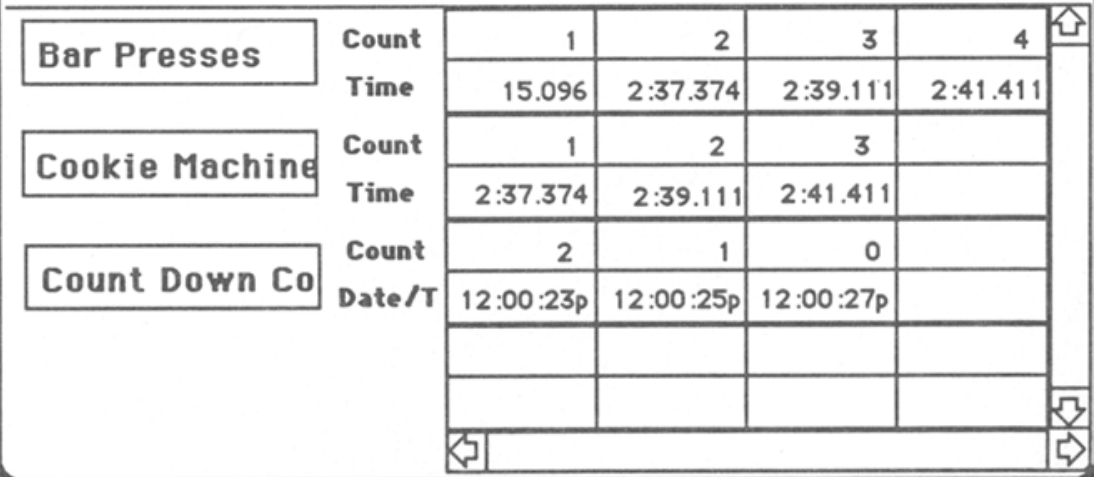

Figure 2. The data display window from a short discrimination learning experiment. Data are saved from userdesignated inputs or outputs as well as from selected palette counters or timers. The tab-delimited data files may be transferred to other applications for analysis. In this example, the subject received three reinforcements while the discrimination cue was present. The experiment was then finished, since three was the maximum number possible.

\section{File Edit Timers Counters Data}

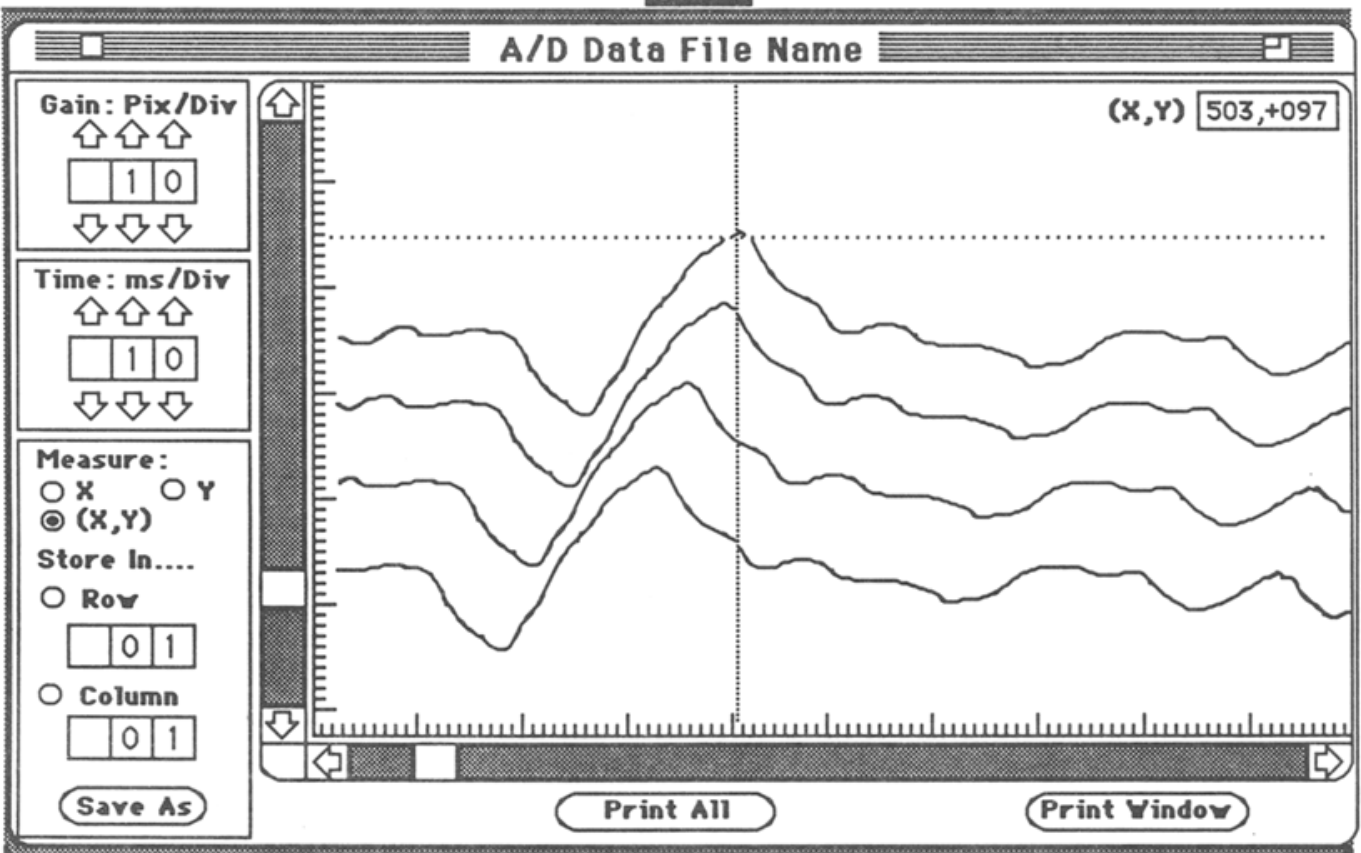

Figure 3. A sample four-channel A/D display. Analog-to-digital data can be displayed and printed. A measurement graticule permits the visualization of any coordinate value, which then may be saved in a specified cell of a tab-delimited file. 


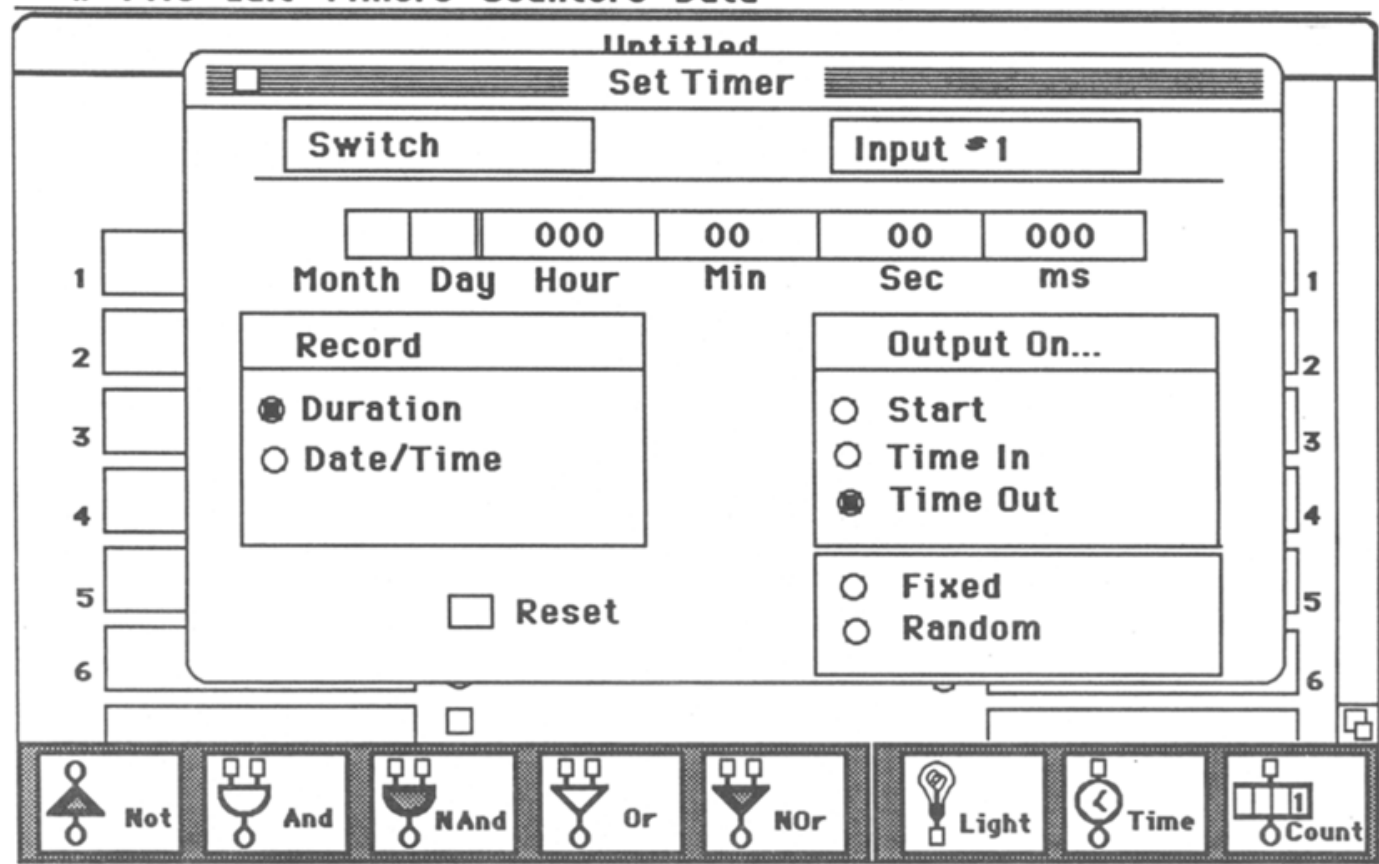

Figure 4. A sample timing window. Timers for input or output boxes, as well as those selected from the palette, can be set to operate in various modes. Timer data can be saved appearing in the data display window. Similar functions for counting are also available and under easy user control.

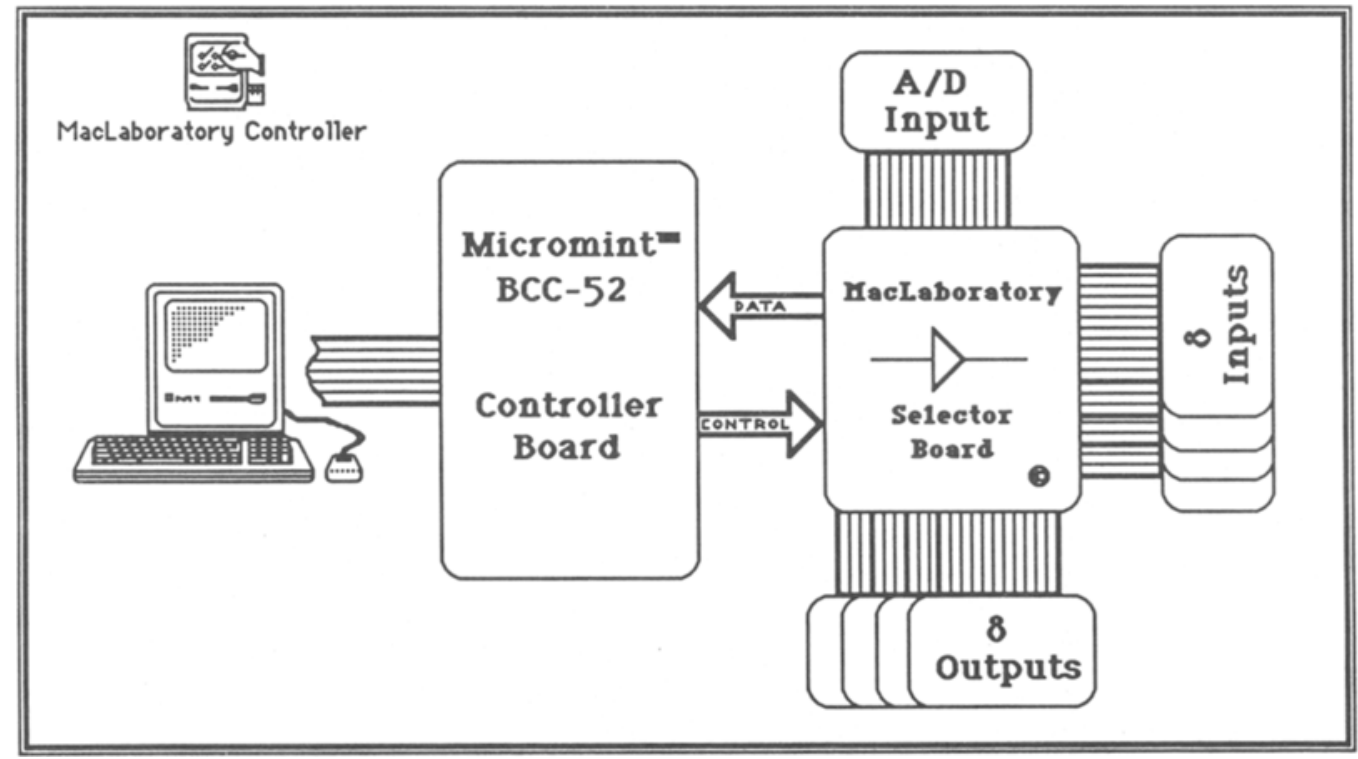

Figure 5. The hardware for the Controller is based on a Micromint BCC-52 Controller Board, a peripheral singleboard computer. User-specified inputs and outputs are individually configured in blocks of eight, depending on the apparatus to be monitored or controlled. Analog-to-digital input can also be supplied. 
BASIC microprocessor, a MacLaboratory EPROM with the BASIC code burned in, 3 parallel ports, and 2 serial ports. It supports a number of expansion boards, including the $\mathrm{BCC} 13 \mathrm{~A} / \mathrm{D}$ converter board. The MacLaboratory Controller also uses a Micromint power supply and terminal back plane. A custom-designed board, a MacLaboratory Selector, provides for Macintosh communication and control of $\mathrm{I} / \mathrm{O}$ modules. The $\mathrm{I} / \mathrm{O} \mathrm{mod}-$ ules (blocks of eight) vary depending on user specifications. Figure 5 illustrates the major components of the system. A typical configuration for output may be one block of relays with $110-\mathrm{V}$ ac 15 -amp rated contacts and one block suitable for 20-50-V dc. Typical input modules may include 4-channel A/D and a block of passive switch closure sensors. More than 32 inputs and 32 outputs are not recommended without upgrades to components of the basic system.

\section{SUMMARY}

MacLaboratory Controller takes advantage of the Macintosh user interface and graphic strengths to supply a readily edited tool for constructing logical relationships between various input and output devices. Data are collected and stored in a manner compatible with the use of other Macintosh packages. These aspects of the Controller solve many of the problems we have seen with similar devices. Specifically, the Macintosh itself does not become dedicated to the Controller, remaining available for other uses, especially data analysis and graphics programs such as Microsoft Excel, with which Controller data files are fully compatible. The software editor creates any number of experimental protocols that are established in the absence of specific programming skills. In our limited experience with the prototype versions thus far, students seem perfectly capable of setting up and using the device. The limiting factor is the students' creativity and imagination in designing experiments, and fortunately not their ability to handle the apparatus.

The cost of the MacLaboratory Controller seems to be less than that of other systems. The basic $16 \mathrm{I} / 0$ switch hardware interface and documentation costs about $\$ 800$, and the software, to be distributed by Kinko's Academic Courseware Exchange, costs about $\$ 40$.

\section{REFERENCES}

Chute, D. L. (1986a). MacLaboratory for psychology: Courseware documentation. Philadelphia: Kinko's Publishing Group.

ChUTE, D. L. (1986b). MacLaboratory for psychology: General experimental psychology with Apple's Macintosh. Behavior Research Methods, Instruments, \& Computers, 18, 205-209.

ChUTE, D. L., \& DANIEL, R. S. (1986a). MacLaboratory for psychology: Instructor's notes. Philadelphia: Kinko's Publishing Group.

ChUTE, D. L., \& DANIEL, R. S. (1986b). MacLaboratory for psychology: Laboratory manual. Philadelphia: Kinko's Publishing Group.

Digitry Company, Inc. (1985). Cognitive Testing Station. Medford, MA: Author.

HewETt, T. T. (1986). When every student has a computer: A new perspective on courseware and its development. Behavior Research Methods, Instruments, \& Computers, 18, 188-195.

LEHMAN, R. (1986). Macintosh statistical packages. Behavior Research Methods, Instruments, \& Computers, 18, 177-187.

SCHLICKHEISER, G. (1986). Benchtop software: Interfacing scientific equipment. Macintosh in the Health Sciences. Apple Computers, Inc.: Memphis Conference.

Westall, R., Perkey, M. N., \& Chute, D. L. (1986). Accurate millisecond timing on Apple's Macintosh using Drexel's MilliTimer. Behavior Research Methods, Instruments, \& Computers, 18, 307-311. 